\title{
NONLINEAR MODELING OF RF/MICROWAVE CIRCUITS FOR MULTI-TONE SIGNAL ANALYSIS
}

\author{
José Carlos Pedro and Nuno Borges Carvalho \\ e-mail: jcpedro@ieee.org and nborges@ieee.org \\ Instituto de Telecomunicações - Universidade de Aveiro, 3810-193 AVEIRO - Portugal
}

\begin{abstract}
Supported on the theoretical framework of Volterra Series, the present paper discusses nonlinear model extraction procedures currently in use for multi-tone signal analysis, and proposes a methodology that has been successfully applied to small and large-signal distortion regimes of microwave circuits. Good agreement between nonlinear simulation results and laboratory measurements of a medium power amplifier driven with band-limited white noise, validated the authors' approach.
\end{abstract}

\section{INTRODUCTION}

The scarcity of spectrum bandwidth available for present and upcoming wireless telecommunications systems has been entailing an increased demand for more linear circuits, stringent distortion figures of merit and progressively involved laboratory characterization procedures [1]-[3].

Microwave/RF nonlinear CAD/CAE now play a dominant role in the telecommunications equipment design process, because the inherent circuits' complexity, associated with the impossibility of getting exact closed-form solutions of even the simplest circuit responses to nonlinear regimes, obviates any attempt of hand-made calculations. This, in turn, asks for more ingenious simulation techniques, but also accurate nonlinear device models and model extraction procedures, since the analysis results can be, at most, as good as the adopted circuit's representation.

Recent advances in the former of these research fields now allow the determination of most circuits' response to a reasonably wide range of stimuli that span from the most simple single-tone or two-tone excitation, to the more complex digital modulated carriers, multi-carrier signals or even band-limited noise.

The nonlinear device model field has also sensed some recent advances that can be grouped into the levels of model formulation and model extraction procedures. Although there are still some work going on for proposing mathematical black-box models or 2D/3D physics device models [4], [5], it seams that empirical modeling based in equivalent circuit descriptions has, for this time, won the nonlinear device modeling dispute. Such models associate empirical mathematical functions to nonlinear controlled sources (usually voltage dependent current sources) or charges/fluxes (for quasi-static nonlinear capacitors or inductors), which are then embedded in a linear equivalent circuit network. Therefore, nonlinear device model extraction procedure becomes a two-step process, in which, first, the linear equivalent circuit topology is obtained, and then, the parameter sets of the sources or charges (or fluxes) are determined. Despite any time a new device family comes into use, novel equivalent circuit topologies and element extraction methods are proposed, it seams that equivalent circuits of classic devices like the BJT/HBT or the MESFET/HEMT are almost stabilized. So, the research efforts have been progressively directed to the nonlinear element model formulations and their parameter extraction methodologies.

The present paper addresses this problem by using the theoretical framework of Volterra Series to state some basic conditions of nonlinear system identification. Then, the methodologies commonly in use are discussed under that theory, and the author's approach is proposed. That is finally validated by showing good agreement between nonlinear simulation results and laboratory measurements of a medium power amplifier driven with band-limited white noise. 


\section{Theoretical Basis For the Extraction of Dynamic Nonlinear Device Models}

For the sake of simplicity, let us consider a certain class of single-input/single-output dynamic nonlinear systems that can be represented by a Volterra Series. If their input signal, $x_{i}(t)$, is composed of a combination of various sinusoids:

$$
x_{i}(t)=\frac{1}{2} \sum_{q=-Q}^{Q} X_{i q} e^{j \omega_{q} t}
$$

then, their output response, $y_{o}(t)$, can be represented by the following series:

$$
y_{o}(t)=\sum_{n=1}^{\infty} \frac{1}{2^{n}} \sum_{q_{1}=-Q}^{Q} \cdots \sum_{q_{n}=-Q}^{Q} X_{q_{1}} \cdots X_{q_{n}} H_{n}\left(\omega_{q_{1}}, \cdots, \omega_{q_{n}}\right) e^{j\left(\omega_{q_{1}}+\cdots+\omega_{q_{n}}\right) t}
$$

in which the $H_{n}\left(\omega_{q 1}, \ldots, \omega_{q n}\right)$ are the so-called nonlinear transfer functions, NLTF, that completely identify the system up to order $n$. The Volterra Series representation then states that the unique identification of an $n$ 'th order system requires the determination of $n$ NLTFs, which depend on a number of frequencies equal to their order. That is, 1st order NLTF (or linear transfer function) demands for several tests using one sinusoid, 2nd order NLTF demands for several tests using two independent sinusoids, and the complete $n$ 'th order NLTF identification requires the use of $n$ independent sinusoids, or $n$ degrees of freedom.

Expressing that in more usual terms, it means that we cannot rely on simple one-tone tests to extract the 2nd order system behavior, since 2nd harmonic do not contain all information needed, nor we should rely on one-tone or twotone tests to extract even the simplest 3rd order system's characteristics. This has already been widely recognized by the scientific community dedicated to the modeling extraction field, which led to first use one-tone tests in nonlinear regimes, like the so-called large-signal S-parameters [6], and more recently two-tone excitations of large amplitude. Indeed, that has been driven by the need to specify and build what could be assumed as an ideal nonlinear network analyzer [7]-[10]. Unfortunately, expression (2) discourages further attempts in this way since it states that the extraction of an accurate dynamic nonlinear system of $n$ order requires laboratory measurements of system responses to $n$ independent sinusoids. And this seams to be unavoidable, unless it is assumed that the system is mildly nonlinear (and thus requiring only a small number of NLTFs) or it exhibits a rapidly vanishing memory compared to the timescales of signals it is intended to operate.

The first hypothesis should be discarded in general, as it is widely recognized that, e.g., 3rd order Volterra representations are insufficient to describe common amplifiers driven close to, or above, their $1 \mathrm{~dB}$ compression point. It is, thus, only utilized for simulating quasi-linear distortion regimes. The second hypothesis is even more unreasonable as microwave devices are continuously driven to their frequency limits.

There is, however, an alternative that can be explored. Since SPICE-like or harmonic-balance simulators require equivalent circuit models where the elements are either dynamic, but linear, or nonlinear, but quasi-static, the proposed way could be to first wipe out the device from its memory by extracting the linear reactive elements, and then simply extract the reminding memoryless nonlinear model descriptions of the dependent sources or charges.

For the concrete case of two-port nonlinear devices, like microwave/RF transistors, that could be implemented following the approach described in Werthof et al. [11]. There, the linear sub-network was considered as a four-port, described, as in Fig. 1, by a set of linear parameters, e. g. a 4x4 [Y] matrix, in which the 1st and 2nd ports are the access ports, and the 3 rd and 4 th are connections to the nonlinear four-pole. 


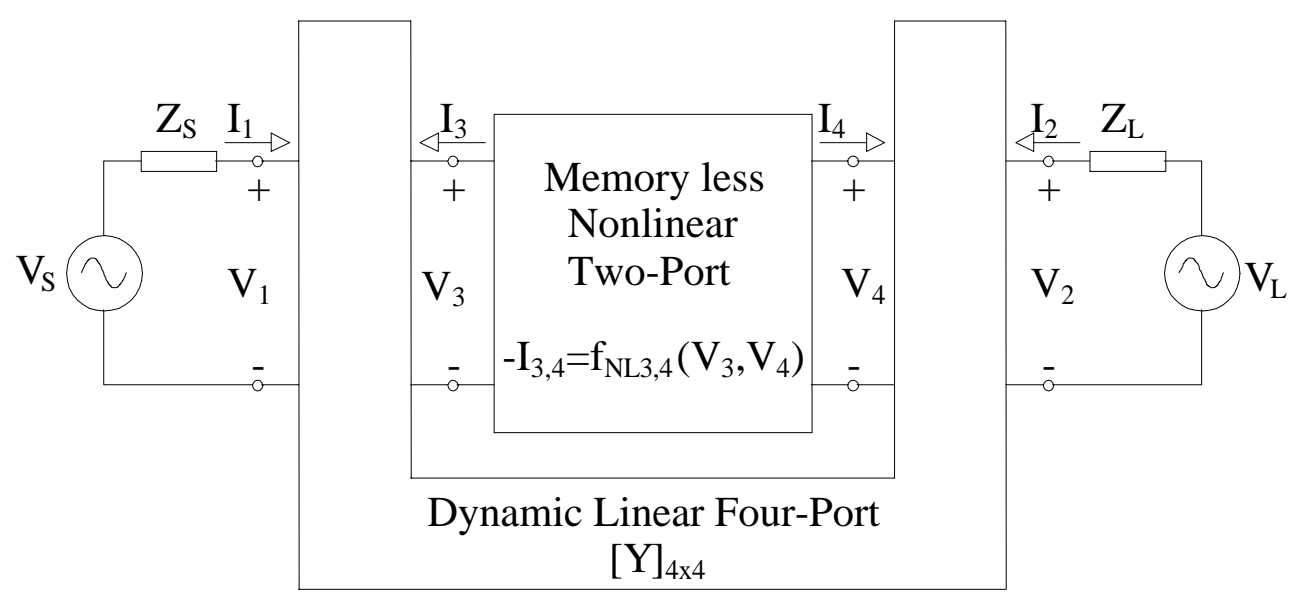

Fig. 1 - Memoryless nonlinear two-port embedded in a linear dynamic circuit.

Since it is assumed that the required linear equivalent circuit extraction procedure has been previously performed, the $4 \times 4[\mathrm{Y}]$ matrix is uniquely known, as are the 1st order linear transfer functions of the nonlinear sub-network. Thus, it is always possible to deduce $\left(I_{3}, I_{4}, V_{3}, V_{4}\right)$ from the access currents and voltages $\left(I_{1}, I_{2}, V_{1}, V_{2}\right)$, and the nonlinear two-port becomes de-embedded.

\section{ILLUSTRATIVE MODEL EXTRACTION EXAMPLE}

For extracting the memoryless nonlinear model descriptions, several considerations must also be taken into account, which will determine the selection of the optimum functional descriptions and the sets of measurements to be performed. In particular, if multi-tone distortion simulations are desired, then a good mathematical function is necessary that is able to approximate, not only the overall device $\mathrm{I} / \mathrm{V}$ characteristics, but also their first three derivatives. That is especially important when usual approximation routines (like minimum squares) or more involved approaches (like neural networks) are used [12].

Our best results were obtained by beginning with a study of the detailed device characteristics from physics simulations. From that, we proposed an approximating function [13], [14], and then extracted the model parameters from DC and small-signal S-parameter data (0'th and 1st order model characteristics), but also from 2nd and 3rd order derivatives obtained from 2 nd and 3rd order harmonic and intermodulation distortion (2nd and 3rd order model characteristics) [15], [16].

Fig. 2 represents the equivalent circuit model extracted for our sample device - a general-purpose GaAs MESFET - using the now classic method of Dambrine [17]. 


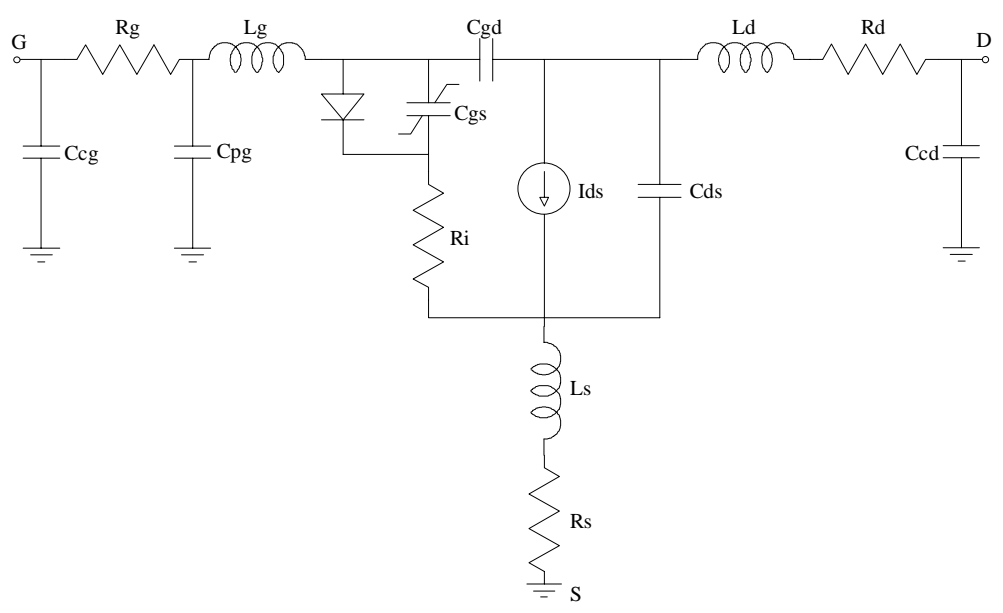

Fig. 2 - GaAs MESFET equivalent circuit model used in the simulations and for deembedding the nonlinear current and charge nonlinearities.

The adopted nonlinear drain-source current model and gate-source charge model are shown in (3-6) and (7), respectively,

$$
I d s(V g s, V d s)=\beta\left\lfloor u+\ln \left(e^{u}+e^{-u}\right) \mid \tanh (\alpha V d s)\right.
$$

where

$$
\begin{aligned}
& u=\frac{1}{2}(h(V g s)-C) \quad \text { (4) } \quad h(V g s)=A(1-d(V g s)) \quad \text { (5) and } \quad d(V g s, V d s)=\sqrt{\frac{V b-V g s}{V p o+\gamma V d s}} \\
& C g s(V g s)=C_{g s 0}+\frac{C_{g s m}}{d(V g s)}[1+\tanh (u)]
\end{aligned}
$$

while Fig. 3 and Fig. 4 present a comparison between the correspondent extracted and model predicted 1st, 2nd and 3rd order derivatives.
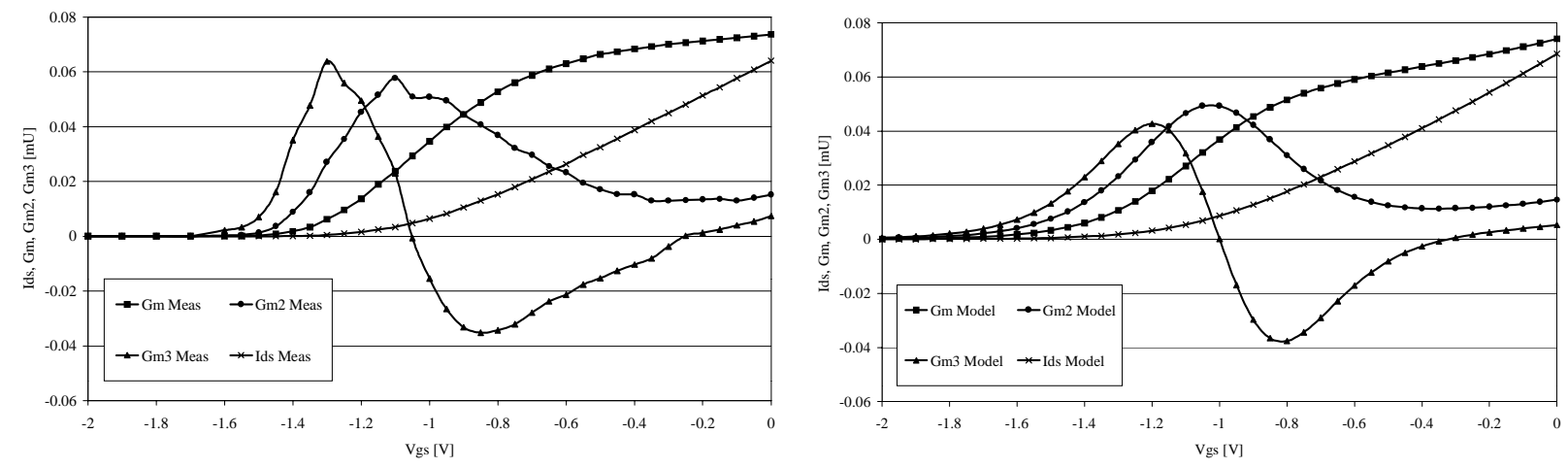

Fig. 3 - Measured and modeled drain source current, and its derivatives, Gm, Gm2 and $G m 3$. 

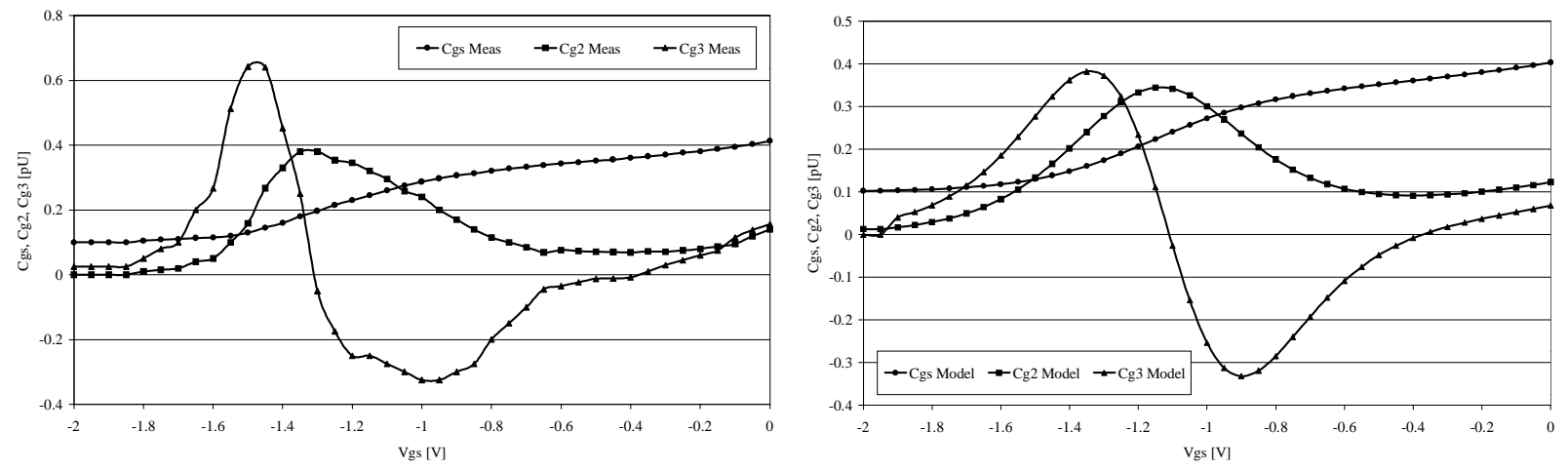

Fig. 4 - Measured and modeled gate source capacitance, and its derivatives $\mathrm{Cgs}, \mathrm{Cg} 2, \mathrm{Cg} 3$.

\section{Nonlinear DeVice Model Validation}

In order to validate the proposed model extraction methodology, a microwave amplifier was then built around our GaAs MESFET and tested under common one-tone, two-tone and also very demanding band-limited white noise excitations. Fig. 5 represents a comparison between measured and simulated Adjacent Channel Power Ratio, ACPR, power sweep data, corresponding to this latter stimulus. Fig. 6, depicts the whole spectrum of one of these power points where not only the adjacent-channel distortion, but also the co-channel distortion is shown. The distortion measurements were made by the authors' Co-Channel Distortion Ratio, CCPR, measurement set-up [2], and the simulations were obtained with the extracted model inserted in an in-house developed artificial frequency mapping based harmonic-balance simulator [18].

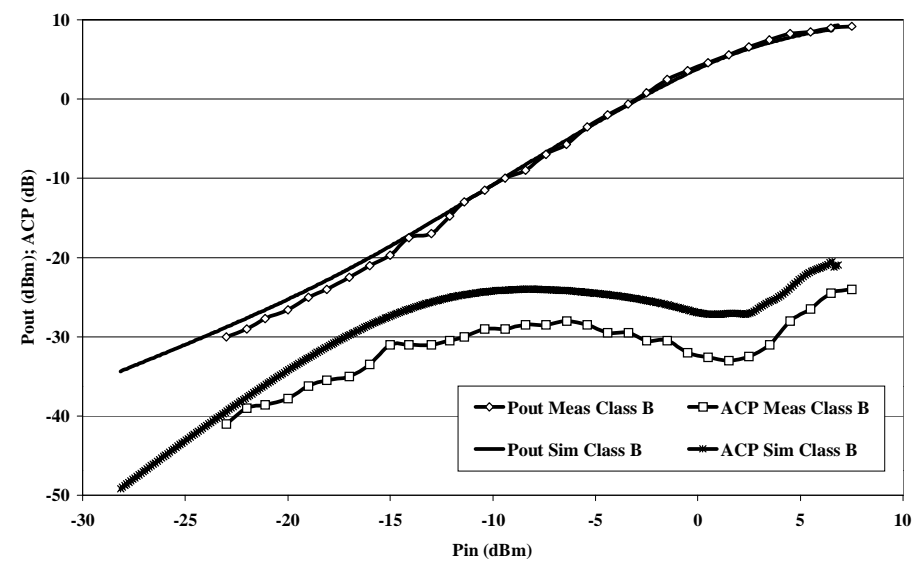

Fig. 5 - Measured and Simulated ACPR vs input power sweep 


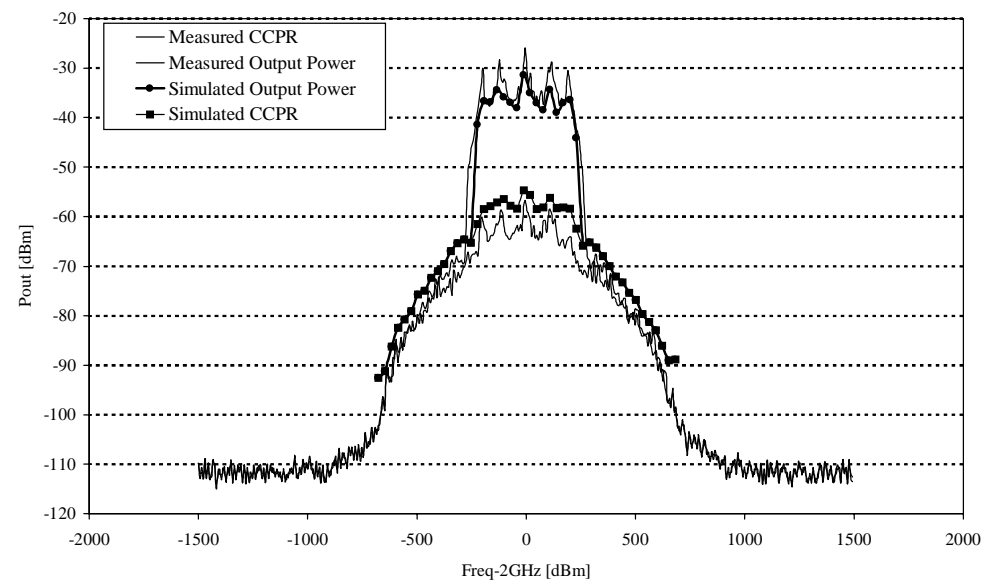

Fig. 6 - Measured and Simulated ACPR and CCPR

A close agreement between the two sets of data is observed in both small and large-signal regimes, which validates the modeling approach in a fairly wide range of the amplifier operation modes.

\section{CONCLUSIONS}

This paper presented a well-defined methodology for the extraction of nonlinear device models. It starts by first deembedding the memoryless nonlinear elements from the linear sub-circuit network of the equivalent circuit. Then, it proposes convenient approximating functions for the nonlinearities, and extracts their parameter sets from largesignal DC I/V, small-signal S parameters and intermodulation measurements. The validity of that approach was shown by comparing measured and simulated distortion characteristics of a microwave amplifier driven by bandlimited white noise.

\section{REFERENCES}

[1] J. C. Pedro and N. B. Carvalho, "On the Use of Multi-Tone Techniques for Assessing RF Components' Intermodulation Distortion", IEEE Trans. on Microwave Theory and Tech., vol. MTT-47, pp.2393-2402, Dec. 1999.

[2] J. C. Pedro and N. B. Carvalho, "Evaluating Co-channel Distortion Ratio in Microwave Power Amplifiers", IEEE Trans. on Microwave Theory and Tech., vol. MTT-49, pp.1777-1784, Oct. 2001.

[3] $\mathrm{H}$. Ku, W. Woo and S. Kenney, "Carrier to Interference Ratio Prediction of Nonlinear RF Devices", Microwave Journal, Vol.44, nº 2, pp.154-164, Feb. 2001.

[4] D. Root, "A Measurement Based FET Model Improves CAE Accuracy", Microwave Journal, pp.126-139, Sep. 1991.

[5] R. Pantoja, M. Howes, J. Richardson, and C. Snowden, C.M., "A Large-Signal Physical MESFET Model for Computer-Aided Design and Its Applications“, IEEE Trans. on Microwave Theory and Tech., vol. MTT-37, pp $2039-2045$, Dec. 1989.

[6] K.M. Johnson, "Large Signal GaAs MESFET Oscillator Design", IEEE Trans. on Microwave Theory and Tech., pp. 217-227, Mar. 1979.

[7] Nonlinear Network Measurement System - Technology Update, Hewlett-Packard Co., Santa Rosa, CA, Dec. 1997.

[8] T. Broeck and J. Verspecht, "Calibrated Vectorial Nonlinear-Network Analyzers", IEEE Intern. Microwave Theory and Tech. Symposium Digest., pp.1069-1072, San Diego, May 1994. 
[9] J. Lekey, A. Patterson and J. Stewart, "A Vector Corrected Waveform and Load Line Measurement System for Large Signal Transistor Characterization", IEEE Intern. Microwave Theory and Tech. Symposium Digest, pp.1243-1246 Orlando, May 1995.

[10] J. Verspecht, P. Debie, A. Barel and L. Martens, "Accurate on Wafer Measurement of Phase and Amplitude of the Spectral Components of Incident and Scattered Voltage Waves at the Signal Ports of a Nonlinear Microwave Device", IEEE Intern. Microwave Theory and Tech. Symposium Digest, pp.1243-1246, Orlando, May 1995.

[11] A. Werthof, F. Raay and G. Kompa, "Direct Nonlinear FET Parameter Extraction Using Large-Signal Waveform Measurements", IEEE Microwave and Guided Wave Lett., vol. 3, pp.130-132, Mar. 1993.

[12] J. A. Garcia, A. Tazón, A. Mediavilla, I. Santamaria, M. Lázaro, C. J. Pantaleón and J. C. Pedro, "Modeling MESFET's and HEMT's Intermodulation Distortion Behavior Using a Generalized Radial Basis Function Network", Intern. Journal of RF and Microwave Computer-Aided Engineering, vol. 9, pp.261-276, May 1999.

[13] J. C. Pedro and J. Perez, "A Novel GaAs FET Model for Intermodulation Analysis in General Purpose Harmonic-Balance Simulators", 23rd European Microwave Conference Proc., pp.714-716, Madrid, Set. 1993.

[14] J. C. Pedro, "A Physics-Based MESFET Empirical Model", IEEE Intern. Microwave Theory and Tech. Symposium Digest, pp.973-976, San Diego, May 1994.

[15] J. C. Pedro and J. Perez, "Accurate Simulation of GaAs MESFET's Intermodulation Distortion Using a New Drain-Source Current Model", IEEE Trans. on Microwave Theory and Tech., vol. MTT-42, pp.25-33, Jan. 1994.

[16] J. A. Garcia, A. M. Sanchéz, J. C. Pedro, N. B. Carvalho, A. T. Puente and J. L. Garcia, "Characterizing the Gate-to-Source Nonlinear Capacitor Role on GaAs FET IMD Performance", IEEE Trans. on Microwave Theory and Tech., vol. MTT-46, pp.2344-2355, Dec. 1998.

[17] G. Dambrine, A. Cappy, F. Helicore and E. Playez, "A New Method for Determining the FET Small-Signal Equivalent Circuit", IEEE Trans. on Microwave Theory and Tech., vol. MTT-36, pp.1151-1159, 1988.

[18] J. C. Pedro and N. B. Carvalho, "Efficient Harmonic Balance Computation of Microwave Circuits' Response to Multi-Tone Spectra", 29th Eurpoean Microwave Conference Proc., vol. I, pp.103-106, Munchen, Oct. 1999. 